\title{
THE LOCAL RESOLVENT SET OF A LOCALLY LIPSCHITZIAN TRANSFORMATION IS OPEN
}

\author{
E. LEE MAY, JR.
}

\begin{abstract}
The purpose of this paper is to prove that if $p$ is a point of a complex Banach space $H$ at which the nonlinear transformation $T$ on $H$ is locally Lipschitzian, then the local resolvent set of $T$ at $p$ is open.
\end{abstract}

Denote by $H$ a complex Banach space with nondegenerate point-set $S$ and norm $\|\cdot\|$ and by $p$ a point of $S$. Denote by $I$ the identity transformation on $H$ and by $T$ a not necessarily linear transformation from a subset $D(T)$ of $S$ into $S$. If $D(T)=S$ and $T$ is bounded and linear, then the resolvent set $\rho(T)$ of $T$ is open [1, pp. 86-89]. Since in this case the local resolvent set $\rho_{p}(T)$ of $T$ at $p$ [2, pp. 212, 213] is $\rho(T)$, this means that $\rho_{p}(T)$ is open. In [2, Theorem 2, pp. 213-215] the openness of $\rho_{p}(T)$ is extended to the case where $T$ is not necessarily linear but continuously Fréchet differentiable on an open set containing $p$ and $H$ is finite-dimensional. The following theorem establishes the fact that neither the differentiability of $T$ nor the finite dimensionality of $H$ is necessary to the openness of $\rho_{p}(T)$.

THEOREM. If $T$ is locally Lipschitzian at $p$, then $\rho_{p}(T)$ is open.

Proof. Denote by $a$ a member of $\rho_{p}(T)$ and by $\left(\delta_{a}, \varepsilon_{a}\right)$ a positive-number pair such that $[2, \mathrm{pp} .212,213]$

(1) $a I-T$ is $1-1$ on the ball $R_{p}\left(\delta_{a}\right)$ with center $p$ and radius $\delta_{a}$;

(2) the ball $R_{(a I-T) p}\left(\varepsilon_{a}\right) \subseteq(a I-T)\left(R_{p}\left(\delta_{a}\right)\right)$;

(3) $\left(a I-\left.T\right|_{R_{p}\left(\delta_{a}\right)}\right)^{-1}$ is Lipschitzian on $R_{(a I-T) p}\left(\varepsilon_{a}\right)\left(\left.T\right|_{R_{p}\left(\delta_{a}\right)}\right.$ is the restriction of $T$ to $R_{p}\left(\delta_{a}\right)$ ).

Properties (1)-(3) permit us to denote by $(r, M)$ a positive-number pair such that $\|(a I-T) x-(a I-T) y\| \geqq M\|x-y\|$ whenever $\{x, y\} \subseteq R_{p}(r)$. Let $A$ $=a I-\left.T\right|_{R_{p}\left(\delta_{a}\right)}$, and denote by $\left|A^{-1}\right|$ the least nonnegative number $B$ such that $\left\|A^{-1} x-A^{-1} y\right\| \leqq B\|x-y\|$ whenever $\{x, y\} \subseteq R_{A p}\left(\varepsilon_{a}\right)$. Finally, let

$$
c=\min \left\{1 /\left(2\left|A^{-1}\right|\right), M, \varepsilon_{a} / 3, \delta_{a} /\left[\left|A^{-1}\right|\left(2+\delta_{a}\right)\right], r /\left[\left|A^{-1}\right|(2+r)\right]\right\},
$$

and suppose that $b$ is a complex number such that $|a-b|<c$. The remainder of the proof will be devoted to showing that $b$ is in $\rho_{p}(T)$. then

Let $\delta_{b}=\min \left\{r, \delta_{a}\right\}$ and $\varepsilon_{b}=\min \left\{\varepsilon_{a} / 2, c\right\}$. If each of $x$ and $y$ is in $R_{p}\left(\delta_{b}\right)$,

Received by the editors November 5, 1974.

AMS (MOS) subject classifications (1970) Primary 47H99; Secondary 47H10.

Key words and phrases. Local resolvent set, nonlinear transformation, locally Lipschitzian, successive approximation, Banach space. 


$$
\begin{aligned}
\|(b I- & T) x-(b I-T) y \| \\
& =\|b x-T x+a x-a x+a y-a y-b y+T y\| \\
& =\|(a I-T) x-(a I-T) y-(a-b)(x-y)\| \\
& \geqq\|(a I-T) x-(a I-T) y\|-|a-b|\|x-y\| \\
& \geqq(M-|a-b|)\|x-y\| \text { since } \delta_{b} \leqq r .
\end{aligned}
$$

Since $|a-b|<c \leqq M$, we have that $M-|a-b|>0$. Thus $b I-T$ is $1-1$ on $R_{p}\left(\delta_{b}\right)$ and $\left(b I-\left.T\right|_{R_{p}\left(\delta_{b}\right)}\right)^{-1}$ is Lipschitzian on $(b I-T)\left(R_{p}\left(\delta_{b}\right)\right)$. It remains to be shown that $R_{(b I-T) p}\left(\varepsilon_{b}\right) \subseteq(b I-T)\left(R_{p}\left(\delta_{b}\right)\right)$.

Denote by $y$ a point of $R_{(b I-T) p}\left(\varepsilon_{b}\right)$. To show that $y$ is in $(b I-T)\left(R_{p}\left(\delta_{b}\right)\right)$, we shall prove that the restriction of the transformation $A^{-1}((a-b) I+y)$ to $R_{p}\left(\delta_{b}\right)$ has a fixed point. The technique used is successive approximation.

Define a sequence $u$ as follows. Let $u_{0}=p$. Then

$$
\begin{aligned}
\left\|(a-b) u_{0}+y-A p\right\| & =\|(a-b) p+y-(a I-T) p\| \\
& =\|y-(b I-T) p\| \\
& <\varepsilon_{b}<\varepsilon_{a} \text { by choice of } \varepsilon_{b} .
\end{aligned}
$$

Thus $(a-b) u_{0}+y$ is in $R_{A p}\left(\varepsilon_{a}\right)$. Since $R_{A p}\left(\varepsilon_{a}\right) \subseteq A\left(R_{p}\left(\delta_{a}\right)\right)$ by (2), let $u_{1}$ $=A^{-1}\left((a-b) u_{0}+y\right)$. Thus

$$
\begin{aligned}
\left\|u_{1}-u_{0}\right\| & =\left\|A^{-1}\left((a-b) u_{0}+y\right)-A^{-1} A p\right\| \\
& =\left\|A^{-1}((a-b) p+y)-A^{-1} A p\right\| \\
& \leqq\left|A^{-1}\right|\|(a-b) p+y-A p\| \\
& <\left|A^{-1}\right| \varepsilon_{b} \leqq\left|A^{-1}\right| c \quad \text { by choice of } \varepsilon_{b} .
\end{aligned}
$$

Furthermore,

$$
\begin{aligned}
\left\|(a-b) u_{1}+y-A p\right\| & =\left\|(a-b) u_{1}-(a-b) u_{0}+(a-b) u_{0}+y-A p\right\| \\
& \leqq|a-b|\left\|u_{1}-u_{0}\right\|+\left\|(a-b) u_{0}+y-A p\right\| \\
& <|a-b|\left|A^{-1}\right| c+\varepsilon_{b}<\left|A^{-1}\right| c^{2}+c .
\end{aligned}
$$

By definition of $c$ we have that $\left|A^{-1}\right| c \leqq 1 / 2$, so $\left|A^{-1}\right| c^{2}+c \leqq(3 / 2) c$ $\leqq(3 / 2)\left(\varepsilon_{a} / 3\right)<\varepsilon_{a}$. Thus $(a-b) u_{1}+y$ is in $R_{A p}\left(\varepsilon_{a}\right)$, so denote by $u_{2}$ the point $A^{-1}\left((a-b) u_{1}+y\right)$. Therefore

$$
\begin{aligned}
\left\|u_{2}-u_{1}\right\| & =\left\|A^{-1}\left((a-b) u_{1}+y\right)-A^{-1}\left((a-b) u_{0}+y\right)\right\| \\
& \leqq\left|A^{-1}\right||a-b|\left\|u_{1}-u_{0}\right\| \\
& <\left(\left|A^{-1}\right| c\right)^{2} \quad \text { since }|a-b|<c \text { and }\left\|u_{1}-u_{0}\right\|<\left|A^{-1}\right| c .
\end{aligned}
$$

This implies the following:

$$
\left\|u_{2}-p\right\| \leqq\left\|u_{2}-u_{1}\right\|+\left\|u_{1}-p\right\|<\left(\left|A^{-1}\right| c\right)^{2}+\left|A^{-1}\right| c .
$$


Finally, we have

$$
\begin{aligned}
\left\|(a-b) u_{2}+y-A p\right\| & =\left\|(a-b) u_{2}-(a-b) u_{1}+(a-b) u_{1}+y-A p\right\| \\
& \leqq|a-b|\left\|u_{2}-u_{1}\right\|+\left\|(a-b) u_{1}+y-A p\right\| \\
& <c\left(\left|A^{-1}\right| c\right)^{2}+c \sum_{i=0}^{1}\left(\left|A^{-1}\right| c\right)^{i} \\
& =c \sum_{i=0}^{2}\left(\left|A^{-1}\right| c\right)^{i} .
\end{aligned}
$$

For the inductive step, suppose that $m$ is an integer not less than 2 and that $u_{0}, u_{1}, \ldots, u_{m}$ has the following properties:

(4) if $k$ is an integer in $[1, m]$, then

$$
\left\|u_{k}-u_{k-1}\right\|<\left(\left|A^{-1}\right| c\right)^{k} \quad \text { and } \quad\left\|u_{k}-p\right\|<\sum_{i=1}^{k}\left(\left|A^{-1}\right| c\right)^{i}
$$

(5) if $k$ is an integer in $[0, m]$, then

$$
\begin{aligned}
& \left\|(a-b) u_{k}+y-A p\right\|<c \sum_{i=0}^{k}\left(\left|A^{-1}\right| c\right)^{i} ; \\
& u_{0}=p \text { and } u_{k}=A^{-1}\left((a-b) u_{k-1}+y\right)
\end{aligned}
$$

for each integer $k$ in $[1, m]$.

Since $\left|A^{-1}\right| c \leqq 1 / 2$, we have that $c \sum_{i=0}^{m}\left(\left|A^{-1}\right| c\right)^{i} \leqq 2 c \leqq 2\left(\varepsilon_{a} / 3\right)<\varepsilon_{a}$. Thus $(a-b) u_{m}+y$ is in $R_{A p}\left(\varepsilon_{a}\right)$, which is a subset of the domain of $A^{-1}$ by (2); so let $u_{m+1}=A^{-1}\left((a-b) u_{m}+y\right)$. Then

$$
\begin{aligned}
\left\|u_{m+1}-u_{m}\right\| & =\left\|A^{-1}\left((a-b) u_{m}+y\right)-A^{-1}\left((a-b) u_{m-1}+y\right)\right\| \\
& \leqq\left|A^{-1}\right||a-b|\left\|u_{m}-u_{m-1}\right\| \\
& <\left|A^{-1}\right| c\left(\left|A^{-1}\right| c\right)^{m} \text { by (4) } \\
& =\left(\left|A^{-1}\right| c\right)^{m+1} .
\end{aligned}
$$

In addition,

$$
\begin{aligned}
\left\|u_{m+1}-p\right\| \leqq & \left\|u_{m+1}-u_{m}\right\|+\left\|u_{m}-p\right\| \\
< & \left(\left|A^{-1}\right| c\right)^{m+1}+\sum_{i=1}^{m}\left(\left|A^{-1}\right| c\right)^{i} \\
& \quad \text { by the preceding inequality and (4) } \\
& =\sum_{i=1}^{m+1}\left(\left|A^{-1}\right| c\right)^{i} .
\end{aligned}
$$

Finally,

$$
\begin{aligned}
\left\|(a-b) u_{m+1}+y-A p\right\| & \leqq|a-b|\left\|u_{m+1}-u_{m}\right\|+\left\|(a-b) u_{m}+y-A p\right\| \\
& <c\left(\left|A^{-1}\right| c\right)^{m+1}+c \sum_{i=0}^{m}\left(\left|A^{-1}\right| c\right)^{i} \\
& =c \sum_{i=0}^{m+1}\left(\left|A^{-1}\right| c\right)^{i} .
\end{aligned}
$$


This completes the inductive step and defines a sequence $u$ with the following properties:

(7) if $n$ is a positive integer, then

$$
\left\|u_{n}-u_{n-1}\right\|<\left(\left|A^{-1}\right| c\right)^{n} \quad \text { and } \quad\left\|u_{n}-p\right\|<\sum_{i=1}^{n}\left(\left|A^{-1}\right| c\right)^{i} ;
$$

(8) if $n$ is a nonnegative integer, then

$$
\begin{aligned}
& \left\|(a-b) u_{n}+y-A p\right\|<c \sum_{i=0}^{n}\left(\left|A^{-1}\right| c\right)^{i} \\
& u_{0}=p \quad \text { and } \quad u_{n}=A^{-1}\left((a-b) u_{n-1}+y\right)
\end{aligned}
$$

for each positive integer $n$.

Since $\left|A^{-1}\right| c \leqq 1 / 2$, we know that $\left\{\sum_{i=0}^{n}\left(\left|A^{-1}\right| c\right)^{i}\right\}_{n=0}^{\infty}$ is convergent and, hence, $u$ is Cauchy. Since $H$ is complete, denote by $x$ the sequential limit of $u$.

To show that $x$ is in $R_{p}\left(\delta_{b}\right)$ and $(b I-T) x=y$, let us first prove that $(a-b) x+y$ is in $R_{A p}\left(\varepsilon_{a}\right)$. If $d>0$ and $t$ is a positive integer such that $\left\|x-u_{t}\right\|<d / c$, we see that

$$
\begin{aligned}
\|(a-b) x+y-A p\| & \leqq|a-b|\left\|x-u_{t}\right\|+\left\|(a-b) u_{t}+y-A p\right\| \\
& <c(d / c)+c \sum_{i=0}^{t}\left(\left|A^{-1}\right| c\right)^{i} \quad \text { by }(8) \\
& <d+2 c \\
& \leqq d+2\left(\varepsilon_{a} / 3\right) \quad \text { by the choice of } c .
\end{aligned}
$$

Thus $\|(a-b) x+y-A p\| \leqq 2\left(\varepsilon_{a} / 3\right)<\varepsilon_{a}$. This means that, since $A^{-1}$ is continuous by (3), we have

$$
\begin{aligned}
x & =\lim _{n \rightarrow \infty} u_{n}=\lim _{n \rightarrow \infty} A^{-1}\left((a-b) u_{n-1}+y\right) \\
& =A^{-1}\left[\lim _{n \rightarrow \infty}\left((a-b) u_{n-1}+y\right)\right]=A^{-1}\left[(a-b) \lim _{n \rightarrow \infty} u_{n-1}+y\right] \\
& =A^{-1}((a-b) x+y) .
\end{aligned}
$$

Therefore $A x=(a-b) x+y$, so $a x-T x=A x=(a-b) x+y$ and $(b I-T) x=y$.

To complete the proof it must be shown that $x$ is in $R_{p}\left(\delta_{b}\right)$. Since $x$ is the sequential limit of $u$, we know by (7) that $\|x-p\| \leqq \sum_{i=1}^{\infty}\left(\left|A^{-1}\right| c\right)^{i}$, which is $\left|A^{-1}\right| c /\left(1-\left|A^{-1}\right| c\right)$ by the fact that $\left|A^{-1}\right| c<1$. The fact that

$$
c \leqq \delta_{a} /\left[\left|A^{-1}\right|\left(2+\delta_{a}\right)\right]
$$

implies that $\left|A^{-1}\right| c /\left(1-\left|A^{-1}\right| c\right) \leqq \delta_{a} / 2$. Similarly, the choice of $c$ to be not greater than $r /\left[\left|A^{-1}\right|(2+r)\right]$ means that

$$
\|x-p\| \leqq\left|A^{-1}\right| c /\left(1-\left|A^{-1}\right| c\right) \leqq r / 2 .
$$

Thus

$$
\|x-p\| \leqq \min \left\{\delta_{a} / 2, r / 2\right\}<\min \left\{\delta_{a}, r\right\}=\delta_{b},
$$


so $x$ is in $R_{p}\left(\delta_{b}\right)$ and $y$ is in $(b I-T)\left(R_{p}\left(\delta_{b}\right)\right)$. This completes the proof.

An example of the phenomenon described by the Theorem is the transformation $I^{*}$ on the complex numbers, which maps each complex number onto its conjugate. $I^{*}$ is Lipschitzian, hence locally Lipschitzian at each complex number. The local resolvent set of $I^{*}$ at 0 -indeed at any complex number-is the complement in the complex numbers of the unit circle.

The Theorem reveals a further similarity between the global spectrum of a bounded linear transformation and the local spectrum [2, pp. 212, 213] of certain nonlinear transformations. In doing so it heightens one's hope that there is a suitable local analog for nonlinear transformations to the spectral representation theory for bounded linear ones. One of the next questions to be answered in attempting to discover such a theory seems to be the following: Does a locally Lipschitzian transformation (or a continuously differentiable transformation on a non-finite-dimensional space) have a local spectrum? It is my feeling that the answer is affirmative.

\section{REFERENCES}

1. E. R. Lorch, Spectral theory, University Texts in the Mathematical Sciences, Oxford Univ. Press, New York, 1962. MR 25 \#427.

2. E. L. May, Jr., Localizing the spectrum, Pacific J. Math. 44 (1973), 211-218. MR 47 \#089.

Department of Mathematics, Salisbury State College, Salisbury, Maryland 21801 